\title{
A retrospective review of Listeria monocytogenes infection at Tygerberg Children's Hospital, Cape Town, South Africa, from 2006 to 2016: Is empirical ampicillin still indicated after the first month of life?
}

\author{
K B Oppel, MB ChB, DCH (SA), FC Paed (SA); S L Holgate, MB ChB, DCH (UK), FC Paed (SA), Cert Neonatology (SA); \\ H Finlayson, MB ChB, DCH (SA), FC Paed (SA), Cert ID (SA) Paed,
}

Department of Paediatrics and Child Health, Faculty of Medicine and Health Sciences, Stellenbosch University, Cape Town, South Africa

Corresponding author: K B Oppel (kimoppel@yahoo.com)

\begin{abstract}
Background. Ampicillin to treat Listeria monocytogenes (LM) infection is empirically added to the treatment of infants ( $<3$ months) with suspected sepsis or meningitis.

Objectives. In view of limited LM cases, the paucity of South African (SA) data and an ampicillin shortage, our objective was to describe the occurrence of LM infections at Tygerberg Hospital (TBH), Cape Town, with the aim of rationalising the paediatric antibiotic policy. Methods. An 11-year (2006 - 2016) retrospective descriptive study of children ( $<13$ years) from TBH and referral hospitals with a positive blood or cerebrospinal fluid (CSF) culture for LM was conducted.

Results. Of 26 children with positive cultures for LM, 23 (88.5\%) were $<3$ months of age; all were $<10$ days old. Approximately half ( $56.5 \%$, $13 / 23$ ) were born at or referred to TBH. Presentation was on the day of delivery in $46.2 \%$ (6/13), $92.3 \%$ were admitted to the neonatal intensive care unit (NICU), and 61.5\% (8/13) died. Neonates treated at peripheral hospitals were statistically more likely than those treated at TBH to have a CSF culture obtained $(90.0 \%$ v. $30.8 \% ; p=0.005)$, and had higher platelet counts $\left(239 \times 10^{9} / \mathrm{L} \mathrm{v.} 107 \times 10^{9} / \mathrm{L} ; p=0.004\right)$, lower C-reactive protein levels $(64 \mathrm{mg} / \mathrm{L} \mathrm{v} .137 \mathrm{mg} / \mathrm{L} ; p=0.013)$ and a lower mortality rate $(0 \%$ v. $61.5 \% ; p=0.002)$. The incidence of $\mathrm{LM}$ at TBH was $0.04 / 1000$ live births and 2.3/1 000 NICU admissions.

Conclusions. As in other countries, the local neonatal LM incidence is low. Neonates present in the first week of life with severe disease and a high mortality rate. These data support a change in antibiotic policy, in keeping with international guidelines, limiting empirical ampicillin prescription to infants $<1$ month of age.
\end{abstract}

S Afr Med J 2018;108(11):937-943. DOI:10.7196/SAMJ.2018.v108i11.13212

Listeria monocytogenes (LM) infection is rare, but can cause severe infection in pregnant women and during the neonatal period. ${ }^{[1]} \mathrm{LM}$ is inherently resistant to some broad-spectrum antibiotics, including third-generation cephalosporins (e.g. ceftriaxone), which are commonly used as first-line antibiotics to treat suspected infection in the community. ${ }^{[2]}$ For this reason, ampicillin is recommended for the empirical treatment of neonatal sepsis (ampicillin and gentamicin) $)^{[3]}$ and neonatal meningitis (ampicillin and a thirdgeneration cephalosporin). Most treatment protocols recommend that ampicillin be added to a third-generation cephalosporin for the treatment of suspected serious bacterial infection in infants aged $<3$ months presenting with a fever, to ensure that cephalosporinresistant organisms, especially LM, are treated. ${ }^{[4]}$

Two distinct clinical syndromes are seen in neonatal listeriosis. Early-onset infection in the first 7 days of life is associated with overwhelming sepsis and a high mortality rate. Late-onset infection occurring between 7 and 90 days has a lower mortality, but $94 \%$ of patients have meningitis, ${ }^{[5]}$ which is associated with poor neurodevelopmental outcomes. ${ }^{[6]}$

Although the majority of neonatal cases present in the first 7 days of life, many guidelines still recommend additional LM cover up to 3 months of age. ${ }^{[1]}$ A case series from the UK reported 362 infant cases of LM from 1970 to 2013 , including sepsis and meningitis. ${ }^{[7]}$ Of all cases of LM, $97 \%$ occurred in the first 90 days of life; of those occurring within the first 90 days, $99 \%$ presented in the first 30 days.
Only 5 cases occurred in infants aged between 31 and 90 days in this 24 -year period. Similarly, in a recent international review of LM infections, only 5 (of a total of 524) cases of LM were reported in infants aged 1 - 3 months. ${ }^{[1]}$ There was a marked decrease in the incidence of pregnancy-related listeriosis in France from 1984 to 2006 related to the implementation of specific LM control measures at the food production level. ${ }^{[8]}$ Data from 2006 to 2012 in the USA documented 181 cases of bacteraemia with no cases of LM in infants. ${ }^{[9]}$ The World Health Organization recommends initial antibiotic therapy with a penicillin and an aminoglycoside or a third-generation cephalosporin for young infants ( $<60$ days of age) ${ }^{[10]}$ Current South African (SA) recommended guidelines for treatment of acute bacterial meningitis include addition of empirical ampicillin for infants aged $<1$ month of age until LM is excluded, ${ }^{[11]}$ but in a number of centres, including ours, ampicillin is still added empirically to the treatment of suspected sepsis or meningitis in infants aged $<2$ months.

There are limited published data on LM from SA. Two studies looking at paediatric LM were published between 25 and 50 years ago. A study done at Baragwanath Hospital in Johannesburg from August 1977 to April 1978 identified 14 cases of LM, 9 in neonates. All the neonates were aged $<2$ weeks at presentation. Four died, all within 3 days of birth or hospital admission. ${ }^{[12]}$

During a 10-year period at King Edward VIII Hospital in Durban from 1981 to 1990, 9 cases of LM were identified; 6 patients were children, of whom 5 were neonates. Three infants died. ${ }^{[13]}$ 
An isolated outbreak occurred in the Western Cape Province in 2015, where a total of 7 cases were documented at a tertiary hospital. ${ }^{[14]}$ One blood culture from a pregnant woman and two cultures from neonates, both 1 day old, isolated LM. ${ }^{[15]}$

A systematic review of neonatal meningitis in developing countries showed that LM was seldom reported in the developing world, with Gram-negative organisms being more common. This was thought to be due to low availability of common potential food contamination sources. ${ }^{[16]}$

The addition of ampicillin to a thirdgeneration cephalosporin for suspected neonatal sepsis and meningitis in neonates and infants aged $<90$ days seems to be appropriate, as most studies ${ }^{[4,7]}$ show that LM is rare after 30 days of age. The question remains whether the empirical addition of ampicillin to a third-generation cephalosporin cannot be further restricted. There is limited evidence to determine the optimal age at which ampicillin therapy is no longer needed as a part of empirical antibiotic regimens. ${ }^{[4]}$ Data from developed countries suggest that empirical ampicillin therapy is no longer needed after 1 month of age, ${ }^{[17]}$ and there are limited data in Africa to support this practice; however, we have no data regarding the prevalence of LM infection at our institution.

In the first half of 2015, we experienced a shortage of ampicillin at Tygerberg Hospital $(\mathrm{TBH})$, such that its use was restricted to a named-patient basis only and empirical cover for fever without a source was changed to a third-generation cephalosporin, even in the absence of meningitis (unpublished data). This raised a concern about missing LM sepsis and meningitis in neonates and young infants.

In view of the limited number of LM cases seen in other countries and the limited SA data available, we reviewed paediatric and neonatal cases of LM at TBH to describe the demographics, clinical presentation and outcome of these patients. This may help provide evidence upon which to rationalise local use of ampicillin in the future, especially in view of the recent ampicillin shortages.

\section{Methods}

\section{Study setting}

TBH provides secondary- and tertiary-level care to residents of the Eastern Metro region of Cape Town. It serves surrounding subdistricts as well as certain areas outside the Cape Metro region (e.g. Winelands). Residents of Metro West are served by a tertiary paediatric hospital as well as two maternity units providing secondary- and tertiary-level care.

\section{Case identification}

A retrospective descriptive study was conducted from 1 January 2006 to 31 December 2016 , to identify patients aged $<13$ years with laboratory-confirmed LM bacteraemia or meningitis. A review of the National Health Laboratory Service (NHLS) database was undertaken to identify all positive cerebrospinal fluid (CSF) and blood cultures processed by the NHLS at TBH. Data were also obtained from the NHLS central data warehouse, which processes data from the Eastern and Western metro. All positive cultures from children $<13$ years of age and pregnant women were included in the analysis.

\section{Data collection}

The folders of children with positive cultures managed at TBH were reviewed and data were entered anonymously onto a data collection form.

Demographic data collected included date of birth, gender, dates of admission and discharge and residential area. Clinical data included gestational age, birth weight, maternal illness, admission vital signs and initial clinical presentation as well as clinical outcome (discharged or died). Laboratory data included the admission full blood count and C-reactive protein (CRP) level, lumbar puncture results if applicable, and HIV status. Microbiological data included date of sample, site of sample (blood or CSF) and antibiotic sensitivity. Empirical use of antibiotic/s at initiation was noted.

For positive cultures from children not referred to $\mathrm{TBH}$, data on age, date of admission and discharge, residential area and outcome were collected from the Western Cape hospital information system.
For pregnant women, data were collected on age, residential area, antepartum illness (including fever and preterm prelabour rupture of the membranes (PPROM)), gestation, date of initiating antibiotics, date of birth of the baby and outcome of the baby.

To calculate LM incidence, total numbers of admissions and live births were obtained from the TBH Department of Paediatrics and Child Health annual reports (unpublished data), Saving Babies reports (Perinatal Problem Identification Programme $)^{[18-20]}$ and personal hospital sources for the Winelands and Overberg regions.

\section{Data analysis, statistics and ethics approval}

Data were analysed using Stata version 14.2 (StataCorp, USA). Continuous variables were summarised using medians and ranges, and frequencies and associated percentages were used to describe categorical variables. Association between categorical variables was assessed using the $\chi^{2}$ distribution or Fisher's exact test. Differences between distributions of white cell counts by group were evaluated using the Wilcoxon rank-sum test.

The study was approved by the Stellenbosch University Health Research Ethics Committee (ref. no. S16/02/033).

\section{Results}

There were a total of 51 positive cultures for LM at the TBH laboratory over the 11-year study period. Half of the patients $(26 / 51$, $51.0 \%)$ were children aged $<13$ years and were included in our analysis; of these, $23 / 26$ $(88.5 \%)$ were $<3$ months of age. There were no children aged $1-3$ months with positive cultures during this period. Of the patients,

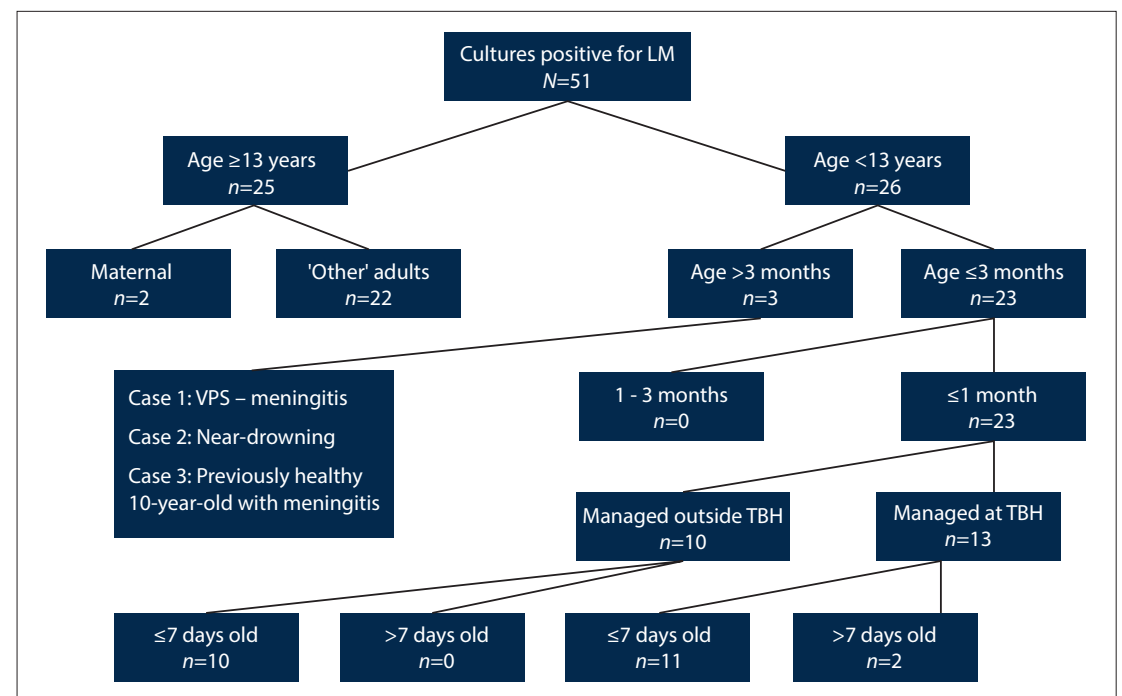

Fig. 1. Patients with cultures (blood and cerebrospinal fluid) positive for LM during the period 1 January 2006 - 31 December 2016. (LM = Listeria monocytogenes; VPS = ventriculoperitoneal shunt; $T B H=$ Tygerberg Hospital.) 


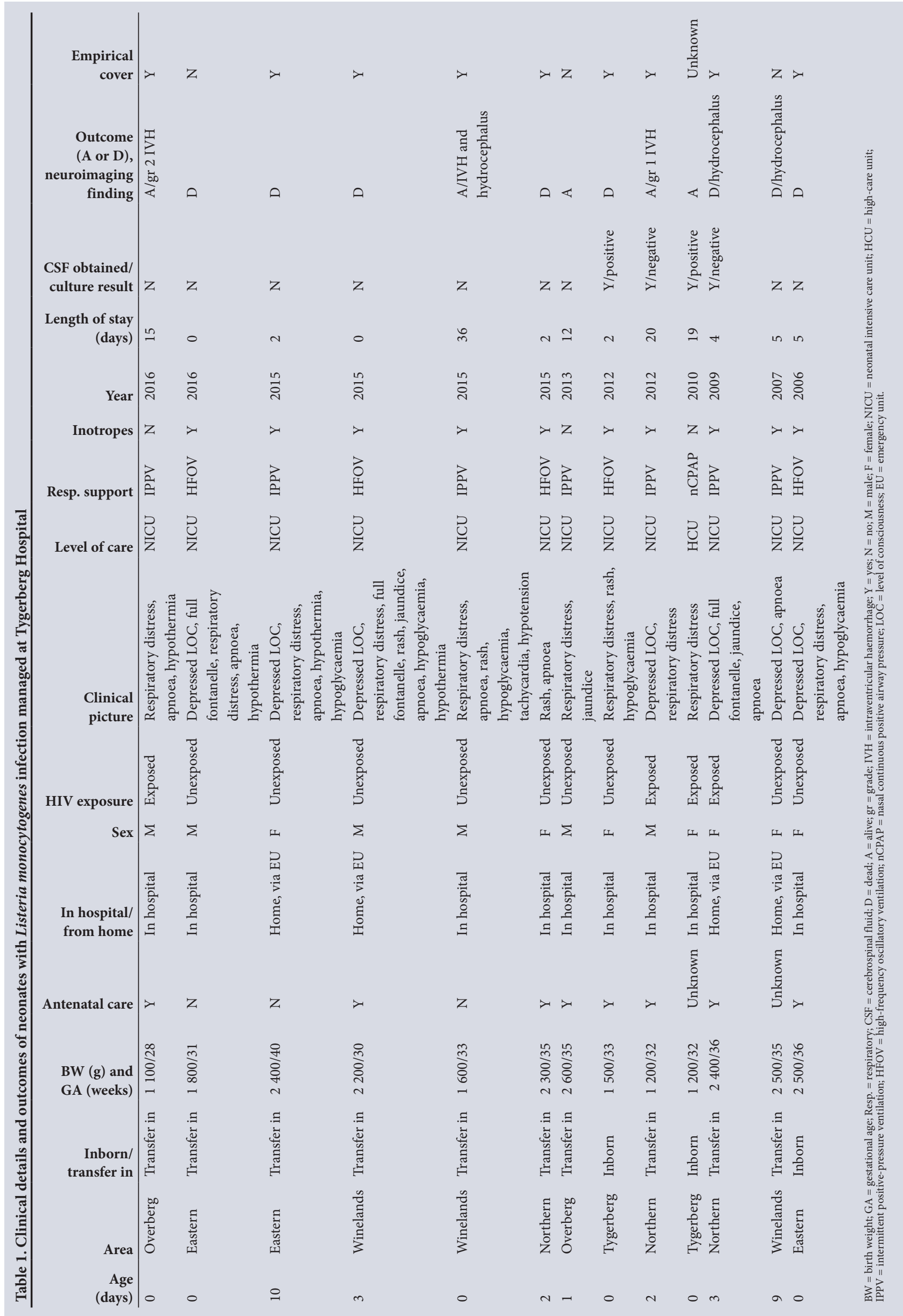


$13 / 23(56.5 \%)$ were managed at $\mathrm{TBH}$ and $10 / 23(43.5 \%)$ at peripheral hospitals (Fig. 1).

One folder was unavailable for review, but laboratory and admission and discharge data were entered for this neonate.

The clinical details and outcomes of the neonates born at or referred to TBH are summarised in Table 1. Cases were evenly distributed over the 11 years, except for 2015 when there were 4 cases. Only $3 / 13(23.1 \%)$ were born at TBH. There was an equal sex distribution (6/13 (46.2\%) male). The majority (12/13, 92.3\%) of neonates were born preterm ( $<37$ weeks' gestation), the median birth weight was $2.2 \mathrm{~kg}$ (interquartile range (IQR) 1.5 - 2.4), and 4/13 (30.8\%) were HIV-exposed; of these, 1 was HIV-infected (positive HIV polymerase chain reaction). The majority of the mothers $(8 / 11$, $72.7 \%$ ) had attended antenatal care during their pregnancy. Eleven neonates (84.6\%) presented within 7 days after birth, nearly half (6/13, $46.2 \%)$ on the day of delivery. Neonates presented with severe disease; 9/13 (69.2\%) presented with respiratory distress and/or apnoea and $7 / 13(53.8 \%)$ with a depressed level of consciousness. Other features noted on admission included a rash $(4 / 13,30.8 \%)$, jaundice $(3 / 13$, $23.1 \%)$, a full fontanelle $(3 / 13,23.1 \%)$ and hypoglycaemia (blood glucose $<2.6 \mathrm{mmol} / \mathrm{L})(5 / 13,38.5 \%)$. The $4 / 13$ neonates $(30.8 \%)$ who presented with hypothermia (temperature $<35.5^{\circ} \mathrm{C}$ ) were transferred from peripheral hospitals. Most patients (12/13, 92.3\%) required intensive care admission and invasive ventilation. The remaining patient required nasal continuous positive airway pressure therapy and high-care admission. Ten neonates (76.9\%) needed inotropic support.

Table 2. Laboratory data for the neonates managed at Tygerberg Hospital

\begin{tabular}{ll}
\hline Investigation & Result \\
\hline Blood glucose $(\mathrm{mmol} / \mathrm{L})$, median $(\mathrm{IQR})$ & $3.9(1.5-6.2)$ \\
White cell count $\left(\times 10^{9} / \mathrm{L}\right)$, median $(\mathrm{IQR})$ & $9.9(3.7-21.9)$ \\
Haemoglobin $(\mathrm{g} / \mathrm{dL})$, median $(\mathrm{IQR})$ & $14.0(12.7-15.2)$ \\
Platelet count $\left(\times 10^{9} / \mathrm{L}\right)$, median $(\mathrm{IQR})$ & $107(66-190)$ \\
$\mathrm{CRP}(\mathrm{mg} / \mathrm{L})$, median $(\mathrm{IQR})$ & $137(107-156)$ \\
$\mathrm{CSF}$ culture obtained, $n(\%)$ & $4 / 13(30.8)$ \\
CSF positive, $n(\%)$ & $2 / 4(50.0)$ \\
CSF polymorphs $(/ \mu \mathrm{L}))$, median $(\mathrm{IQR})$ & $24.5(15.8-29.3)$ \\
CSF lymphocytes $(/ \mu \mathrm{L}))$, median $(\mathrm{IQR})$ & $104(54.5-244.5)$ \\
CSF glucose $(\mathrm{mmol} / \mathrm{L})$, median $(\mathrm{IQR})$ & $2.7(2.6-3.8)$ \\
CSF protein $(\mathrm{g} / \mathrm{L})$, median $(\mathrm{IQR})$ & $1.7(1.6-1.9)$ \\
$\mathrm{IQR}=$ interquartile range; $\mathrm{CRP}=\mathrm{C}$-reactive protein; CSF = cerebrospinal fluid.
\end{tabular}

Eight neonates $(61.5 \%)$ died, with their median length of stay being 2.5 days (IQR $1.5-4.3$ ). Of the 13 patients, 3 had hydrocephalus, presumed to be secondary to Listeria meningitis. The median length of stay for the neonates who were discharged alive was 19 days (IQR 15 - 20). Of the 13 patients, 9 (69.2\%) received empirical Listeria cover.

Table 2 sets out the laboratory data for the neonates described in Table 1. Of the 13 patients, $11(84.6 \%)$ had an elevated CRP level (>10 mg/L) on presentation. Only 4/13 (30.8\%) had a CSF sample taken; of the samples, $2 / 4$ (50.0\%) cultured LM.

CSF findings revealed a lymphocyte predominance, normal glucose and mildly elevated CSF protein.

Table 3 shows the comparison between the neonates managed at TBH and those managed outside TBH. Both groups of neonates presented early $(p=0.66)$ and within 10 days of birth.

The neonates managed at peripheral hospitals were more likely than the TBH group to have a CSF sample obtained for culture $(p=0.005)$. Although the infants managed at TBH were more likely to have a positive CSF culture, this difference did not reach statistical significance. White cell counts and haemoglobin concentrations were similar between the two groups ( $p=0.952$ and $p=0.072$, respectively), but the median platelet count was significantly lower $\left(107 \times 10^{9} / \mathrm{L}\right.$ (IQR $66-190)$ v. $\left.239 \times 10^{9} / \mathrm{L}(\mathrm{IQR} 167-254) ; p=0.004\right)$ and the CRP level significantly higher (137 mg/L (IQR 107 - 156) v. $64 \mathrm{mg} / \mathrm{L}$ (IQR 36 - 97); $p=0.01)$ in the TBH group. The neonates managed at TBH (including those who died) had a shorter duration of stay (5.0 days (IQR 2 - 15) v. 20.5 days (IQR 14.5 - 23); $p=0.013$ ) and significantly higher mortality ( $61.5 \%$ v. $0 \% ; p=0.002)$. For those who survived, the duration of stay was similar at the two locations (19 days (IQR 15 - 20) v. 20.5 days (IQR 14.5 - 23); $p=0.9$ ).

There were too few patients to statistically compare those with LM born at TBH with those transferred in from outside hospitals. Key findings are tabulated in Table 4 . The two groups appeared to be similar with regard to clinical presentation and disease severity, but neonates born at TBH had a lower median glucose level on admission and a lower CRP level compared with the other group.

There were 25 cases of LM in individuals $\geq 13$ years of age. Only $3 / 25$ (12.0\%) occurred in pregnant women, in 2009, 2015 and 2016, respectively. Of these women, all had booked for antenatal care, 2 had a history of fever, none had PPROM and 2 were HIV-positive. Only 1 required respiratory support and high care. One mother gave birth to twins.

In all the pregnant women blood cultures were positive for LM, and all were started on empirical LM cover during the 2 weeks

Table 3. A comparison between neonates managed at TBH and those managed at peripheral hospitals

\begin{tabular}{|c|c|c|c|}
\hline & Managed at TBH $(N=13)$ & Managed outside TBH $(N=10)$ & $p$-value \\
\hline Age at presentation (days), median (IQR) & $0(0-3)$ & $0.5(0-1)$ & 0.6622 \\
\hline Weight $(\mathrm{kg})$, median (IQR) & $2.2(1.5-2.4)$ & $\mathrm{n} / \mathrm{a}$ & - \\
\hline CSF culture obtained, $n$ (\%) & $4 / 13(30.8)$ & $9 / 10(90.0)$ & 0.005 \\
\hline CSF positive, $n(\%)$ & $2 / 4(50.0)$ & $2 / 9(22.2)$ & 0.320 \\
\hline Length of stay (days) (IQR) & $5(2-15)$ & $20.5(14.5-23)$ & 0.013 \\
\hline Outcome death, $n(\%)$ & $8 / 13(61.5)$ & $0 / 10(0)$ & 0.002 \\
\hline Blood glucose (mmol/L), median (IQR) & $3.9(1.5-6.2)$ & $\mathrm{n} / \mathrm{a}$ & \\
\hline White cell count $\left(\times 10^{9} / \mathrm{L}\right)$, median (IQR) & $9.9(3.7-21.9)$ & $8.0(4.0-14.8)$ & 0.952 \\
\hline Haemoglobin (g/dL), median (IQR) & $14.0(12.7-15.2)$ & $16.0(14.4-17.8)$ & 0.072 \\
\hline Platelet count $\left(\times 10^{9} / \mathrm{L}\right)$, median $(\mathrm{IQR})$ & $107(66-190)$ & $239(167-254)$ & 0.004 \\
\hline CRP (mg/L), median (IQR) & $137(107-156)$ & $64(36-97)$ & 0.013 \\
\hline
\end{tabular}


Table 4. A comparison between neonates born at TBH and those transferred to TBH

\begin{tabular}{|c|c|c|}
\hline & Born at $\mathrm{TBH}(N=3)$ & Transferred in $(N=10)$ \\
\hline Age (days), median (IQR) & $0(0-0)$ & $1.5(0-3)$ \\
\hline Presented in hospital, \% & 100 & 60.0 \\
\hline Outcome death, $n(\%)$ & $2 / 3(66.7)$ & $6 / 10(60.0)$ \\
\hline Blood glucose (mmol/L), median (IQR) & $1.4(1.2-1.6)$ & $4.9(2.4-6.3)$ \\
\hline White cell count $\left(\times 10^{9} / \mathrm{L}\right)$, median $(\mathrm{IQR})$ & $6.7(4.6-8.3)$ & $12.72(4.4-23.0)$ \\
\hline Platelet count $\left(\times 10^{9} / \mathrm{L}\right)$, median $(\mathrm{IQR})$ & $190(148.5-390)$ & $102.5(60-146)$ \\
\hline CRP (mg/L), median (IQR) & $87(43.5-97)$ & $142(116.8-163)$ \\
\hline
\end{tabular}

Table 5. Listeria monocytogenes infection incidence rates per area, 2006 - 2016

\begin{tabular}{ll}
\hline Area & Incidence, $\boldsymbol{n} / \boldsymbol{N}(\mathbf{1 ~ 0 0 0 )}$ \\
\hline TBH: Live births & $3 / 75297(0.04)$ \\
TBH neonatal high care: Admissions & $3 / 22614(0.13)$ \\
TBH NICU: Admissions & $12 / 5134(2.3)$ \\
Winelands: Live births & $6 / 67383(0.09)$ \\
Overberg: Live births & $3 / 30056(0.1)$ \\
Metro East: Live births & $14 / 309204(0.05)$ \\
Metro West: Live births & $27 / 390162(0.07)$ \\
TBH = Tygerberg Hospital; NICU = neonatal intensive care unit.
\end{tabular}

prior to delivery. All their infants were empirically managed with ampicillin following delivery, although none were clinically unwell and all had negative blood cultures. Two of the infants had negative CSF cultures, and lumbar puncture was not performed in the other two. All the mothers and their infants were discharged alive.

There were 3 cases of LM in children aged 3 months - 13 years. In 2009 LM was cultured from the CSF of a 9-month-old child with a ventriculoperitoneal shunt infection, and in 2013 a 2-year-old child admitted following near-drowning subsequently cultured LM on blood culture. Both patients developed signs of meningitis and sepsis and were diagnosed with LM infection $~ 2$ weeks after admission. The third case, in 2015, was in a previously healthy 10-year-old who was diagnosed with LM meningitis. Immunodeficiency screens in this patient were all negative.

We identified an additional 84 cases of LM in adults and children in the Metro West region between 2006 and 2016 via the NHLS central data warehouse. Of these cases, 29 were in children aged $<13$ years, with 27 occurring in infants aged $<3$ months. All these patients were aged $<1$ month of age and all cultures were taken between 0 and 2 days of age.

The incidence rates of LM for TBH and surrounding areas including Metro West are summarised in Table 5. Incidences across all areas were low except for the TBH NICU.

\section{Discussion}

The objective of this study was to determine whether empirical ampicillin is indicated outside the neonatal period. There were only 13 cases of LM infection in infants aged $<3$ months (all in neonates $<1$ month old) at TBH during the 11-year study period, while only 3 sporadic cases occurred beyond 3 months of age. These results support the restriction of ampicillin for the treatment of severe infection, including meningitis, to neonates $\leq 1$ month of age, especially when stocks of ampicillin are limited.

The incidence of neonatal LM at TBH from 2006 to 2016 $(0.04 / 1000$ live births) is in keeping with the low incidence rates in developed countries, namely the UK (0.05/1 000 live births), The
Netherlands $(0.01 / 1000)$ and the USA $(0.09 / 1000) .^{[1]}$ The incidence of LM causing severe infection may be an underestimate, as not all neonates are treated in district health facilities where blood and CSF cultures are available. Infants who died before contact with healthcare facilities would also not have been among the cases captured. Empirical treatment of sepsis before obtaining cultures may also decrease the laboratory yield of LM.

The increased number of cases in 2015 could be explained by the increased cases seen in the Cape Metro at this time. ${ }^{[15]}$

In our study the presence of maternal fever or PPROM was not very well documented, as in the Baragwanath Hospital study. ${ }^{[12]}$ All the neonatal cases presented within the first 10 days of life with features of severe sepsis, similar to the presenting symptoms of infants described in the Baragwanath and Durban studies. ${ }^{[12,13]}$ Similarly, in a neighbouring Western Cape health region (Western Metro), all the cases of LM occurred within the first 2 days of life. The majority of our cases also presented in the early neonatal period (within the first week), with only two cases of late-onset infection occurring between 7 and 10 days. In our study, 50\% of the neonates who underwent lumbar puncture had a CSF culture positive for LM. This is in contrast to $100 \%$ in the Durban study. ${ }^{[13]}$ The significance of this finding is limited, however, as in our study CSF was not obtained from the majority of the neonates because they were considered too unstable to tolerate a lumbar puncture. The CSF of patients in our study revealed a lymphocyte predominance, in contrast to the polymorphonuclear predominance in the Durban study. ${ }^{[13]}$ The age pattern did not differ between the neonates treated in regional hospitals who did not require referral to the tertiary hospital and those who were managed at TBH (all were $<10$ days of age); however, the former were more likely to have a CSF culture obtained, although only $22.2 \%$ were culture-positive.

In our study, the mortality rate for neonates diagnosed with LM managed at TBH was $62.0 \%$. These neonates had risk factors known to be associated with a poor outcome: clinical presentation within the first few days of life, requiring admission to an intensive care unit, and prematurity. These findings are in keeping with previous studies ${ }^{[5,13]}$ highlighting the mode of presentation and high mortality rate of early-onset infection. ${ }^{[12]}$ The paradox of the zero mortality rate $(0 \%)$ of the neonates treated in regional hospitals in contrast to those treated in the tertiary care hospital can probably be explained by the transfer of severely ill neonates to the tertiary care hospital. HIV infection has been shown to be a risk for LM infection ${ }^{[21,22]}$ and this has been evident in the recent outbreak in SA. ${ }^{[23]}$ In our study, 30.8\% of the infants infected with LM were HIV exposed (one infected); this is two-fold higher than the current HIV prevalence rates of $16-18 \%$ in pregnant women in the Western Cape. ${ }^{[24]}$ Although our numbers are small, the higher HIV prevalence rate in our study may indicate that neonates whose mothers are HIV-positive have an increased risk of LM infection. ${ }^{[23]}$ 
Four of the neonates were admitted from home via the emergency unit in their respective hospitals directly to the TBH NICU. The rest presented soon after delivery, before discharge from hospital, with nearly half (44.4\%) presenting on the day of delivery. None of the neonates treated for LM were admitted via the paediatric medical casualty department or via the neonatal high-care ward that serves neonates referred from regional hospitals. This probably reflects the severity of illness, requiring direct intensive care admission.

Early-onset infection with LM in the first 7 days of life is associated with overwhelming sepsis and a high mortality rate. ${ }^{[5]}$ The typical presentation of late-onset LM is a term neonate born to an asymptomatic mother who presents with nonspecific illness and laboratory indicators of meningitis. ${ }^{[25]}$ The two TBH patients with late-onset infection presented at 9 and 10 days of age and CSF was not obtained from either; however, both died. Other cases that fitted this profile may have been missed if blood or CSF had not been drawn for culture or if cultures were falsely negative.

In an international review of LM cases, only $0.1 \%$ ( 5 out of 524 cases) occurred between 1 and 3 months of age. ${ }^{[1]}$ Together with the results of our study, this supports limiting the use of ampicillin, in combination with a third-generation cephalosporin, to neonates $<1$ month of age suspected of having severe infection and requiring meningitis cover. This would be in keeping with international guidelines and recommendations from other developed countries such as Canada, ${ }^{[26]}$ the USA ${ }^{[27]}$ and The Netherlands. ${ }^{[28]}$ This policy would be appropriate where stocks of ampicillin are limited, as has been experienced in the local health system. For infants and children outside this age range, using a third-generation cephalosporin as a single agent would cover the most common bacteria in this age group. This is currently the drug of choice for children presenting with possible severe infection to clinics run by Integrated Management of Childhood Illnesses ${ }^{[29]}$-trained nurses and to our paediatric emergency centre.

An additional argument for the use of ampicillin in neonates being treated for severe infection is that ampicillin will also cover cephalosporin-resistant Enterococcus. ${ }^{[4]}$ Enterococcus is an uncommon cause of bacteraemia and meningitis and a more common cause of urinary tract infection (UTI). ${ }^{[30]}$ It is also more likely to present in a neonate or infant who has been hospitalised for a long time. ${ }^{[3]}$ In our setting, Enterococcus bloodstream infection usually follows a UTI and is a cause of nosocomial bloodstream infections. ${ }^{[32]}$ We specifically wanted to identify the need for ampicillin in community-acquired infections presenting in the first 3 months of life and therefore did not include enterococcal infections in our data collection and analysis. However, medical staff should be aware of the possible need for additional cover with ampicillin in the setting of UTI and nosocomial infections in young infants.

Subsequent to our study, SA experienced a nationwide Listeria outbreak with a total of 820 laboratory-confirmed cases reported to the National Institute for Communicable Diseases between January 2017 and 23 January 2018. Of the cases 13\% were from the Western Cape, of which 13 were neonates managed at TBH. Outcome data available for 134 cases show a $45 \%$ mortality rate. Similar to the findings in our study, there is an almost equal distribution between adult and neonatal cases, with $39 \%$ of cases being in neonates aged $<28$ days, with $96 \%$ of these patients $<6$ days old. Only $25 \%$ of LM-infected females aged 15 - 49 years were pregnant. ${ }^{[23]}$

\section{Study limitations}

This study has a number of limitations. Firstly, the number of infections caused by LM may have been underestimated, as we included only neonates and children who were culture-positive.
Neonates and children treated for severe infection may have had false-negative cultures for LM, i.e. an insufficient sample or a blood sample for culture drawn after first antibiotic dose. Stillborn fetuses and infants who died before cultures could be taken may also have had a severe LM infection. Secondly, the retrospective nature of the study may have resulted in some cases being missed. These limitations could have led us to underestimate the incidence of infection, but not its clinical presentation.

\section{Conclusions}

Given that none of our cases of LM in neonates over the past 11 years presented after 10 days of age, it would be safe to limit empirical ampicillin prescription to neonates presenting with presumed sepsis at this tertiary care hospital to the first month of life. Similar previous data from other regions of the country support this change in policy, but before the recommendation is accepted nationwide, studies in other tertiary and regional hospitals should be conducted to confirm this finding. In an outbreak situation, as was being experienced in SA at the time of writing, it would be important to consider a change in protocol to provide empirical cover for older children and adult patients presenting with signs and symptoms compatible with LM infection, until the epidemiology of the outbreak is adequately described.

Declaration. This article was written as partial requirement for KBO's MMed (Paediatrics) degree.

Acknowledgements. We thank Maxwell Chirehwa of the Biostatistics Department at Stellenbosch University for his assistance with the statistical analysis and Prof. Robert Gie for all his help, support and encouragement. Author contributions. The research question was conceived by HF and $\mathrm{KBO}$. KBO performed the data collection and was the primary author. HF and SLH contributed editing and supervision of the writing of the manuscript.

Funding. None.

Conflicts of interest. None.

1. Okike IO, Lamont RF, Heath PT. Do we really need to worry about Listeria in newborn infants? Pediatr Infect Dis J 2013;32(4):405-406. https://doi.org/10.1097/INF.0b013e3182867faO

2. Troxler R, von Graevenitz A, Funke G, et al. Natural antibiotic susceptibility of Listeria species: L. grayi, L. innocua, L. ivanovii, L. monocytogenes, $L$. seeligeri and $L$. welshimeri strains. Clin Microbiol Infect 2000;6(10):525-535. https://doi.org/10.1046/.1469-0691.2000.00168.x

3. Gordon RC, Barrett FF, Clark DJ. Influence of several antibiotics, singly and in combination, on the growth of Listeria monocytogenes. J Pediatr 1972;80(4):667-670. https://doi.org/10.1016/S0022$3476(72) 80072-6$

4. Brown JC, Burns JL, Cummings P. Ampicillin use in infant fever. Arch Pediatr Adolesc Med 2002;156(1):27-32. https://doi.org/10.1001/archpedi.156.1.27

Bortolussi R. Listeria monocytogenes infections in neonates. Semin Pediatr Infect Dis 1999;10(2):111Bortolussi R. Listeria monocytogenes infections in neo

118. https://doi.org/10.1016/S1045-1870(99)80042-9

Clauss HE, Lorber B. Central nervous system infection with Listeria monocytogenes. Curr Infect Dis Rep 2008;10(4):300-306. https://doi.org/10.1007/s11908-008-0049-0

7. Herberg JA, Emonts M, Jacobs M, et al. Empirical antibiotic cover for Listeria moncytogenes infection beyond the neonatal period: A time for change? Arch Dis Child 2015;100(5):421-423. https://doi. org/10.1136/archdischild-2014-307860

8. Girard D, Leclercq A, Laurent E, Lecuit M, de Valk H, Goulet V. Pregnancy-related listeriosis in France, 1984 to 2011, with a focus on 606 cases from 1999 to 2011. Euro Surveill 2014;19(38):pii=20909. https://doi.org/10.2807/1560-7917.ES2014.19.38.20909

9. Biondi E, Evans R, Mischler M, et al. Epidemiology of bacteremia in febrile infants in the United States. Pediatrics 2013;132(6):990-996. https://doi.org/10.1542/peds.2013-1759

10. World Health Organization. World Health Report 2005: Make Every Mother and Child Count. Geneva: WHO, 2005:219. http://www.who.int/whr/2005/whr2005_en.pdf (accessed 27 September 2018).

11. Boyles TH, Bamford C, Bateman, $\mathrm{K}$ et al. Guidelines for the management of acute meningitis in children and adults in South Africa. South Afr J Epidemiol Infect 2013;28(1):5-15. https://doi.org/1 children and adults in South Af
$0.1080 / 10158782.2013 .11441513$

12. Jacobs MR, Stein H, Buqwane A, et al. Epidemic listeriosis: Report of 14 cases detected in 9 months. S Afr Med J 1978;54(10):389-392.

13. Lalloo UG, Coovadia YM, Adhikari M, Poyiadji O. Listeria monocytogenes at King Edward VIII 3. Lalloo UG, Coovadia YM, Adhikari M, Poyiadji O. Listeria monocytogenes at King
Hospital, Durban: A 10-year experience, 1981 - 1990. S Afr Med J 1992;81(4):187-189.

14. Listeriosis - an apparent cluster of cases in the Western Cape Province. NICD Commun Dis Commun 2015;14(11):10-11

15. Smith A, Naicker P, Bamford C, et al. Genome sequences for a cluster of human isolates of Listeria monocytogenes identified in South Africa in 2015. Genome Announc 2016;4(2):e00200-16. https://doi. org/10.1128/genomeA.00200-16

16. Furyk JS, Swann O, Molyneux E. Systematic review: Neonatal meningitis in the developing world. Trop Med Int Health 2011;16(6):672-679. https://doi.org/10.1111/j.1365-3156.2011.02750.x 
17. Malley M, Garg A, Monaghan M, Kampmann B. Prescribing amoxicillin for babies up to 3 months of age: Definitely time for change. Arch Dis Child 2016;101(3):294. https://doi.org/10.1136/ of age: Definitely time for
archdischild-2015-310219

18. Pattinson RC. Saving Babies 2008 - 2009: Seventh Report on Perinatal Care in South Africa. Pretoria: Tshepesa Press, 2011.

19. Pattinson RC. Saving Babies 2010 - 2011: Eighth Report on Perinatal Care in South Africa. Pretoria: Tshepesa Press, 2013

20. Pattinson RC, Rhoda N. Saving Babies 2012 - 2013: Ninth Peport on Perinatal Care in South Africa. Pretoria: Tshepesa Press, 2014

21. Charlier C, Perrodeau E. Clinical features and prognostic factors of listeriosis: The MONALISA national prospective cohort. Lancet Infect Dis 2017;17(5):510-519. https://doi.org/10.1016/S1473 3099(16)30521-7

22. Goulet V, Herbert M, Hedberg C, et al. Incidence of listeriosis and related mortality among groups at risk of acquiring listeriosis. Clin Infect Dis 2012;54(5):652-660. https://doi.org/10.1093/cid/cir902

23. Situation Report on Listeriosis Outbreak: 2017 - 2018: NICD latest update. 25 January 2018. http:// www.nicd.ac.za/wp-content/uploads/2018/01/NICD-Situation-update-on-listeriosis-outbreak-SouthAfrica 25-January-2018.pdf (accessed 12 February 2018).

24. Joint United Nations Programme on HIV/AIDS (UNAIDS). Global AIDS Update 2016. Geneva: UNAIDS, 2016. http://www.unaids.org/sites/default/files/media_asset/global-AIDS-update-2016_ en.pdf (accessed 27 September 2018)

25. Posfay-Barbe KM, Wald ER. Listeriosis. Semin Fetal Neonatal Med 2009;14(4):228-233. https://doi. org/10.1016/j.siny.2009.01.006

26. Le Saux N. Guidelines for the management of suspected or confirmed bacterial meningitis in Canadian children over one month. Paediatr Child Health 2014;19(3):147-152. https://doi.org/10.1093/ pch/19.3.147
27. Tunkel AR, Hartman BJ, Kaplan SL, et al. Practice guidelines for the management of bacterial meningitis. Clin Infect Dis 2017;39(9):1267-1284. https://doi.org/10.1086/425368

28. Dutch Working Party on Antibiotic Policy (SWAB). Guidelines on Antibacterial Therapy of Patients with Bacterial Central Nervous System Infection. Nijmegen, Netherlands: SWAB, 2011. https:// with Bacterial Central Nervous System Infection. Nijmegen, Netherlands: SWAB, 2011. https:/ www.swab.nl/swab/cms3.nsf/uploads/54BD4C530402974EC12579C

29. Integrated Management of Childhood Illnesses (IMCI) Manual 2014. Department of Health, Republic of South Africa. http://paediatrics.ukzn.ac.za/Files/2014\%20IMCI\%20CHART\%20BOOKLET\%20 Final.pdf (accessed 27 September 2018)

30. Hassoun A, Stankovic C, Rogers A, et al. Listeria and enterococcal infections in neonates 28 days and younger: Is empiric ampicillin still indicated? Paediatr Emerg Care 2014;30(4):240-243. https://doi. org/10.1097/PEC.0000000000000104

31. Kimberlin D. Meningitis in the neonate. Curr Treat Options Neurol 2002;4(3):239-248. https://doi. org/10.1007/s1 1940-002-0041-1

32. Dramowski A, Madide A, Bekker A. Neonatal nosocomial bloodstream infections at a referral hospital in a middle income country: Burden, pathogens, antimicrobial resistance and mortality. Paediatr Int Child Health 2015;35(3):265-272. https://doi.org/10.1179/2046905515Y.0000000029

Accepted 29 May 2018 